\title{
Por qué China no cuenta con una clase media nueva: cuadros líderes, gerentes y empresarios
}

DOI: $10.32870 /$ mycp.v12i35.326

David S. G. Goodman ${ }^{1}$

\section{Resumen}

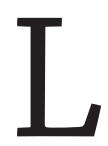

a reforma económica de la República Popular China (RPC) ha visto desde 1980 el surgimiento de nuevas categorías de riqueza y poder. En forma colectiva y coloquial, estas nuevas categorías se conocen como los "nuevos ricos". Se les ha denominado también, tanto fuera como dentro de la RPC, como la nueva clase media (o clases medias) por diferentes razones. Si bien existen buenos motivos para aceptar esta designación, la evidencia de encuestas sobre los nuevos empresarios ricos realizadas desde principios de 1990 en diferentes partes de la RPC (Zhejiang, Shanxi, Qinghai, Sichuan y Hainan) sugiere precaución. En términos de jerarquía de riqueza, estatus y poder, los nuevos ricos representan no sólo un componente significativo de la clase gobernante actual, sino quizás y de manera más importante resaltan las dimensiones de un futuro a largo plazo.

Es claro que desde principios de la década de los ochenta existe un cambio social masivo en la RPC propiciado por el rápido crecimiento económico. Tomar el ejemplo más obvio de creación de riqueza y llevarlo a una escala dramática ha significado que la RPC se haya convertido en algo sin igual. En 1978, China contaba con una sociedad prácticamente igualitaria en términos de ingreso, aunque era, de forma considerable, una igualdad en pobreza. El coeficiente

1. Profesor de Política China y Director del Instituto de Ciencias Sociales en la Universidad de Sydney ORCID http://orcid.org/0000-0003-4965-6177 
Gini, que es una de las medidas estándar de igualdad, en la cual la igualdad está representada como 0 y la desigualdad como 1, fue de 0.46 en 2005 en China, habiéndose convertido en una de las sociedades más desiguales del mundo. La desigualdad regional de ingresos creció rápidamente a partir del principio de autosuficiencia hasta una posición en la cual la jurisdicción a nivel provincial más pobre (Guizhou) representa menos de $8 \%$ del PIB per cápita de la más rica (Shanghai). ${ }^{2}$

Los principales beneficiarios y agentes de este importante crecimiento económico han sido una gran variedad de nuevos empresarios quienes a escala grande y pequeña, a través del comercio minorista, la fabricación y los servicios, han inventado, invertido, poseído y administrado su camino hacia los diferentes grados de riqueza. Desde finales de los años ochenta y principios de los noventa, estos empresarios han sido señalados cada vez con mayor frecuencia como la nueva "clase media" o "clases medias" tanto por los académicos como por los comentaristas de los medios en general fuera de China. ${ }^{3}$ En el proceso queda implícito claramente un paralelismo entre el desarrollo social de China desde finales de los años setenta y las consecuencias de la industrialización europea durante un periodo más largo que inició en el siglo XIX.

Si bien la retórica de la convergencia entre lo que originalmente parecía una divergencia amplia entre los sistemas social y económico se está confrontando, y es un subtema varias veces repetido dentro y fuera de China, el supuesto de la equivalencia entre el caso europeo y el chino también es algo que se presenta fácilmente. Las clases medias no son un concepto sencillo, sino que está compuesto por varios elementos y por sí mismo se considera en ocasiones estratificado: una razón clara es que con frecuencia también se les refiere como clases medias, en plural. Es interesante que las clases medias se ven ahora generalmente en las sociedades industrializadas, a medida que

2. Wang Shaoguang y Hu Angang The Political Economy of Uneven Development: The Case of China M E Sharpe, Armonk, Nueva York, 1999, p. 199ff.

3. Por ejemplo: R. M. Glassman, China in Transition: Communism, Capitalism and Democracy, Nueva York, Praeger, 1991; Gordon White, Jude Howell y Shang Xiaoyuan, In Search of Civil Society: Market Reform and Social Change in Contemporary China, Clarendon Press, Oxford, 1996; David S. G. Goodman, "The New Middle Class", en Merle Goldman y Roderick MacFarquhar (eds.), The Paradox of China's Post-Mao Reforms, Harvard University Press, 1999, pp. 241-261; y Nicholas R. Lardy, “China's Economy: Problems and Prospects", en Footnotes (boletín del Instituto para la Investigación de Políticas Extranjeras del Fondo Marvin Wachman de Estados Unidos para la Educación Internacional), vol. 12, núm. 4, febrero de 2007. 
los sectores grandes y medianos de las jerarquías contemporáneas de riqueza económica, estatus social y poder político se identifican tanto por su consumo y adherencia a un estilo de vida, como en términos socioeconómicos. ${ }^{4}$

Las conceptualizaciones actuales de las clases medias se relacionan con algo ligeramente diferente al surgimiento del concepto de clase media, y sus orígenes, en el contexto europeo. A pesar de que el concepto es complejo por necesidad, se puede reducir ampliamente a dos definiciones generales y determinadas históricamente: la burguesía y (por separado) los administradores del Estado moderno.

A partir del inicio del siglo XIX, la burguesía constituyó una nueva clase media creada por el proceso de la industrialización. Fueron los capitanes de la industria, propietarios de los medios de producción en actividades de extracción, procesamiento y manufactura, que impulsó la industrialización y se convirtieron en la clase media porque no eran ni la arraigada aristocracia, por un lado, ni tampoco los pueblerinos ordinarios, por otro. La industrialización se profundizó hacia finales del siglo XIX y principios del siglo XX sobre la base de empresas económicas y del Estado moderno actual, siendo más compleja y produciendo clases gerenciales y profesionales. Estas dos se convirtieron en las nuevas clases medias debido a que si bien ninguna de ellas poseía el capital ni controlaba el Estado, fungían como una u otra, o ambas, y derivaban sus ingresos y estatus del servicio y la administración en lugar de la propiedad de los medios de producción. ${ }^{5}$

En ambos casos, la importancia de estas nuevas clases medias es tanto política como social y económica. La demanda por ampliar la franquicia y el surgimiento de la democracia liberal durante la primera mitad del siglo XIX en el noroeste de Europa, con frecuencia se consideran como resultados necesarios del surgimiento de la burguesía. La revolución gerencial de la primera mitad del siglo xx es correspondiente al desarrollo de la sociedad masiva, la política masiva y el Estado de bienestar. ${ }^{6}$

4. Richard Robison y David S. G. Goodman, "The new rich in Asia: economic development, social status and political consciousness", en R. Robison y D. S. G. Goodman (eds.), The New Rich in Asia: Mobile phones, McDonald's and middle class revolution, Londres, Routledge, 1996, pp. 1-16.

5. James Burnham, The Managerial Revolution, Greenwood Press, 1972 (reimpresión de la edición original de 1940).

6. William Kornhauser, The Politics of Mass Society, Free Press, Glencoe, 1959; John K. Galbraith, The New Industrial State, Hamish Hamilton, 1967. 
Si bien la RPC ha visto claramente el surgimiento de nuevas categorías de empresarios, la pregunta clave es en qué medida estos nuevos ricos se pueden identificar como el equivalente de las clases medias en otros contextos socioeconómicos anteriores. ${ }^{7}$ Ciertamente existe un discurso más evidente de las clases medias que aparecen en descripciones del cambio social en la República Popular China desde el exterior, así como en las autodescripciones internas. ${ }^{8}$ En cierta medida y desde algunas perspectivas, independientemente de la construcción socioeconómica, el supuesto de un comportamiento de clase media, en especial en cuanto a patrones de consumo, no sólo es razonable, también es algo esperado como una función de la comercialización globalizada. Gucci, Loewe y Louis Vuitton son marcas enfocadas a los consumidores ricos en Shanghai y Beijing, al igual que lo son en Milán, Londres y Nueva York.

Al mismo tiempo, existe espacio para tener precaución por lo mucho que se dice con respecto de los procesos del cambio social en China. La identificación de una conducta de clase media en la China contemporánea no necesariamente implica una ecuación con las anteriores clases medias de otras sociedades y claramente también es necesario aislar las diferencias además de las similitudes. En especial, existen tres aspectos del surgimiento de las nuevas categorías de empresarios ricos en China, mismas que por separado resaltan su relación con el estatus social, la riqueza económica y el poder político, que sugieren ser más bien una parte futura central de la clase gobernante que de una clase media. En cierto sentido, China tuvo una revolución gerencial antes de la revolución burguesa (a pesar de que el curso de la historia no se inició en 1949) con la

7. Margaret M. Pearson, China's New Business Elite: The Political Consequences of Economic Reform, University of California Press, 1997; David L. Wank, Commodifying Communism: Business, Trust, and Politics in a Chinese City, Cambridge University Press, 1999; Doug Guthrie, Dragon in a Three-Piece Suit: The Emergence Capitalism in China, Princeton University Press, 1999; y Bruce Dickson, Red Capitalists: The Party, Private Entrepreneurs, and Prospects for Political Change, Cambridge University Press, 2003.

8. Qin Yan, Zhongguo zhongchan jieji [La clase media china], Zhongguo Jihua Chubanshe, Beijing, 1999; Li Peilin, Zhongguo Shehui Fenceng [Estratificación social en China hoy], Shehui Kexue Wenxian Chubanshe, Beijing, 2004; Zheng Hangsheng, Dangdai Zhongguo Chengshi Shehui [Estructura social de las ciudades en la China contemporánea], Zhongguo Renmin Daxue Chubanshe, Beijing, 2004; Zheng Hangsheng (ed.), Zhongguo Shehui Jiegou Bianhua Qushi Yanjiu [Estudios de las tendencias cambiantes en la estructura social china], Zhongguo Renmin Daxue Chubanshe, Beijing, 2004; Lu Xueyi (ed.), Dangdai Zhongguo Shehui Jieceng Yanjiu baogao [Informe sobre la investigación de las clases sociales en la China Contemporánea], Shehui Kexue Wenxian Chubanshe, Beijing, 2002; Zhou Xiaohong (ed.), Zhongguo zhongchanjieceng diaocha [Encuesta sobre las clases medias chinas], Beijing, Shehui kexue wenxian chubanshe, 2005. 
creación de clases gerenciales y profesionales como parte del desarrollo de un Estado que se modernizaba durante la década de los cincuenta. Ciertamente, los empresarios posteriores a la era de 1978 incluyen no sólo los que se encuentran bien económicamente, sino aquéllos que son sumamente ricos y extra-ricos. Al mismo tiempo, han sido y permanecen diferentes a la burguesía europea de la primera mitad del siglo xIX en la medida en que surgieron y tienen relaciones organizativas cercanas con el sistema político establecido.

En este artículo presento resultados de investigación de campo, que he realizado a partir de los años noventa, sobre las clases medias en China, incluyendo la más reciente sobre mujeres empresarias en Jiaocheng, Qiongshan y Mianyang en un proyecto llevado a cabo con Minglu Chen. El artículo incluye cuatro apartados: la RPC y las clases medias; los empresarios y desarrollo empresarial; los empresarios y riqueza y los empresarios y el partido-Estado. Una conclusión sobre los nuevos empresarios y la clase media cierra el artículo.

\section{La RPC y las clases medias}

Es mucho más fácil considerar a los nuevos ricos posteriores a la China de 1978 como el equivalente a la clase media contemporánea europea o norteamericana, si la modernización se considera únicamente a partir de la era posterior a Mao. Sin embargo, el desarrollo económico sostenido que se ha experimentado desde principios de la década de 1980 no es la primera etapa de la modernización en China. Pese a que la modernización es claramente un concepto controversial, en términos amplios es posible identificar tres eras de industrialización y modernización en la experiencia del siglo xx de China.

La Era Republicana vio intentos sostenidos de modernización en diferentes partes de China, tanto bajo la regla del caudillo como de la influencia 
colonial. ${ }^{9}$ Esto incluyó el desarrollo de las industrias del hierro y el acero, de la minería de carbón a gran escala, de la maquinaria, de la textil, de las instituciones financieras, de los buques y ferrocarriles y una industria del tabaco excepcionalmente grande. Ello en gran medida debido a que esta actividad económica era suministrada, poseída o respaldada externamente, pues desde principios de la década de 1920 algunas de las economías chinas estaban mucho mejor integradas a la economía mundial de lo que sería posteriormente, desde 1937 hasta $1978 .{ }^{10}$ Incluso los condados montañosos que se encuentran a cierta distancia de la costa, como Liaoxian, en la zona norte del interior de China (en las fronteras con las provincias de Shanxi y Hebei), suministraban productos a los mercados externos durante la década de $1930 .{ }^{11}$

Probablemente de mayor importancia para entender la más reciente economía política del cambio, una vez asegurado el nuevo régimen, el establecimiento de la República Popular China desembocó en una industrialización y modernización renovada y sostenida, después de la dislocación que tuvo lugar durante los decenios de 1930 y 1940. Los años transcurridos entre 1952 a 1978 no dejaron de tener problemas económicos; especialmente durante la primera etapa de la década de los sesenta cuando la economía se vio amenazada por una implosión en los albores del Gran Salto, o durante el punto álgido de la Revolución Cultural cuando se vio limitada la producción. No obstante, la economía de la República Popular China logró una tasa global de crecimiento anual de $6 \%$ a lo largo de la era dominada por Mao. ${ }^{12}$

En el corazón de este crecimiento se encontraba el desarrollo de un Estado moderno, incluida la construcción de redes de comunicaciones y el suministro

9. Jonathan D. Spence, The Search for Modem China, Norton and Co, Nueva York, 1990, especialmente p. 325; James E. Sheridan, China in Disintegration: The Republican Era in Chinese History, 1912-1949, The Free Press, Macmillan, Londres, 1975; Donald G. Gillin Warlord Yen Hsi-shan in Shansi Province 1911-1949, Princeton University Press, Princeton, New Jersey, 1967; Robert A. Kapp, Szechwan and the Chinese Republic: Provincial Militarism and Central Power, 1911-1938, Yale University Press, 1973; Christian Henriot, Shanghai 1927-1937: Municipal Power, Locality, and Modernization, University of California Press, 1993.

10. Philip Richardson, Economic Change in China 1800-1950, Cambridge University Press, 2005.

11. Xu Guosheng y Chen Ninghua (eds.), Shanxi xianqu jingji fazhan shilue [Descripción histórica del desarrollo económico de los Condados y Regiones de Shanxi], Taiyuan, Shanxi jingji chubanshe, 1992, 'Zuoquanxian' ('condado de Zuoquan') pp. 556-557. Liaoxian fue renombrado Zuoquan en 1942.

12. Ma Hong et al. (ed.), Quangua gesheng, zizhiqu, zhixiashi lishi tongji ziliao huibian 1949-1989 [Colección de estadísticas históricas de las provincias chinas, regiones autónomas y municipios dirigidos centralmente, 1949-1989], Beijing, Zhongguo tongji chubanshe, 1990, p. 6. 
de una estructura de educación, salud y bienestar, un tanto más enfocada en las áreas urbanas que en las rurales. Ciertamente la burguesía y en general los propietarios a gran escala de bienes se vieron desposeídos durante una serie de campañas diseñadas para asegurar la socialización de los medios de producción durante los años 1952-1955. ${ }^{13} \mathrm{Al}$ mismo tiempo, los años cincuenta vieron el crecimiento de las ocupaciones a nivel gerencial y profesional al servicio del nuevo Estado, en su administración y gestión económica, y quienes en diferentes formas eran observados como las clases medias centrales de la República Popular China: claramente no es un término que se hubiera usado en la República Popular China en una era dominada por las fórmulas ideológicas del pensamiento marxista-leninista-maoísta. Fueron, y en gran medida lo siguen siendo, tanto social como individualmente, aquéllos que se convirtieron en instrumentos del Estado y el capital en contraposición a los que toman decisiones estratégicas o los productores de la línea frontal.

El establecimiento y desarrollo de un nuevo Estado requerían una amplia base de ejecutivos. Si bien se daba considerable atención a los cuadros militares y a los cuadros de líderes que poblaban el partido-Estado, la nueva burocracia también engendró un gran ejército de funcionarios y administradores de menor nivel, conocidos en chino como "petty cadres" (xiao ganbu). ${ }^{14}$ En gran medida estos pequeños cuadros eran la esencia del estado burocrático establecido por el Partido Comunista Chino (PCC). Los altos cargos militares generalmente eran ocupados por aquellos que se habían unido al PCC y a la revolución antes del final de la Guerra de la Resistencia de 1945. A medida que creció el movimiento comunista durante la Guerra Civil, estas personas lograron cargos de liderazgo que después fueron transferidos al nuevo Estado y posteriormente, en 1949, a medida que se expandió el PCC del norte y noreste de China para ocupar todo el país, se convirtieron en la nueva clase dirigente. ${ }^{15}$ Bajo su liderazgo, el partido-Estado tenía la responsabilidad no

13. John Gardner, "The Wu-fan Campaign in Shanghai: A Study in the Consolidation of Urban Control", en A. Doak Barnett (ed.), Chinese Communist Politics in Action, University of Washington Press, 1969, p. 477.

14. A Doak Barnett, Cadres, Bureaucracy and Political Power in Communist China, Columbia University Press, 1967.

15. Frederick C. Teiwes, Provincial Party Personnel in Mainland China, East Asia Institute, Columbia University, 1967; David S. G. Goodman, "The Provincial First Party Secretary in the People's Republic of China, 1949-1978: A Profile”, en The British Journal of Political Science, vol. 10, núm. 1, enero 1980, p. 39; Zhiyue Bo, Chinese Provincial Leaders: Economic Performance and Political Mobility since 1949, M E Sharpe, Nueva York, 2002. 
sólo de la administración del Estado y reglamentación de su vida social, también proporcionaba servicios sociales y de bienestar y operaba la economía. La producción económica estaba dirigida completamente por el Estado, a pesar de que tan sólo una parte de ésta era gestionada de manera inmediata mediante departamentos gubernamentales. Los funcionarios, administradores y gerentes que ocupaban los cargos en esta amplia burocracia inicialmente fueron contratados de manera local durante los años cincuenta y a medida que esta práctica continuó se complementó con la asignación de estudiantes universitarios, a punto de graduarse, a cargos en cualquier lugar de la República Popular China. ${ }^{16}$

En forma paralela y en ocasiones sobrepuesta con la burocracia del socialismo de Estado, el nuevo Estado también aseguró el futuro desarrollo de las profesiones, mismas que se expandieron mucho más allá de los inicios de la Era Republicana. Los maestros, médicos e ingenieros eran los más numerosos pues a medida que el Estado se modernizaba, ampliaba sus actividades y alcance. Al mismo tiempo, la década de los cincuenta vio también el surgimiento de rutas de carrera para otros profesionales, incluidos abogados y economistas, aunque altamente politizados. Estas clases medias intelectuales fueron precisamente aquellas criticadas durante la Revolución Cultural a fines de los 1960 por convertirse en la "novena categoría corrupta de contrarrevolucionarios". Muchos perdieron sus cargos y posesiones, al menos temporalmente, hasta que en los 1970 fueron enviados al interior o a la producción de línea frontal para "reeducación" y algunos sufrieron hasta abuso físico. ${ }^{17}$

Después de 1978, el impulso de la República Popular China por mayor industrialización y modernización atrajo y en cierta medida dependió de estas clases medias gerenciales y sus familias. Al igual que la reputación de los cuadros líderes que fueron removidos durante la Revolución Cultural, y en ocasiones las personas mismas, poco a poco la mayoría de ellos fueron reinstalados durante los años setenta, de manera que la reputación de la clase media volvió a resurgir. El proceso de restaurar sus cargos en la sociedad y el empleo ya se había iniciado mucho antes de la muerte de Mao, en septiembre

16. "The standard sociological examination of urban China in the Mao-dominated era", en Martin King Whyte y William L Parish, Urban Life in Contemporary China, The University of Chicago Press, 1984.

17. L. Esherik, P. Piekowiez, A. Walder (eds.), China's Cultural Revolution as History, Stanford University Press, 2006; R. MacFarquhar y M. Schoenhals, Mao's Last Revolution, Bellknap Press, Nueva York, 2006. 
de 1976. Sin embargo, la década de los setenta vio una restauración más completa y explícita, en ocasiones incluyendo un pago para resarcir los daños. La educación y la capacitación en general volvieron a formar parte de la agenda del partido-Estado a medida que buscaba el crecimiento económico. De esta manera, los distintos tipos de conocimientos y experiencias profesionales de nuevo fueron movilizados para los objetivos de desarrollo de China.

\section{Empresarios y desarrollo empresarial}

La identificación de las clases medias profesionales y gerenciales en la RPC no significa necesariamente que los empresarios surgidos como resultado de las reformas económicas introducidas desde 1978 no sean también clases medias. Sin embargo, esto debe ser examinado más de cerca, tanto en términos del desarrollo de China como de un contexto comparativo más amplio.

En especial, atraen la atención las características específicas de los nuevos empresarios como clases medias, así como su relación con las clases profesionales y gerenciales. Claramente, estas dos amplias categorías sociales pueden tener varias cosas en común, siendo una de ellas este conjunto compartido de aspiraciones de estilo de vida, entre las cuales se encuentran vivir en una casa propia, tener un coche, asegurar una educación privada para los hijos y ser capaz de participar en actividades de ocio, que pueden incluir vacaciones fuera del país. ${ }^{18}$ También, es posible que los miembros de estas clases medias profesionales y gerenciales anteriores a 1978 se hayan transformado en un nuevo estilo de empresarios durante los años ochenta. Al mismo tiempo, el reconocimiento de que existe y permanece una clase media profesional y gerencial patrocinada por el Estado sugiere que estos nuevos empresarios quizás no sean diferentes a las clases medias, pero que el proceso de formación y conceptualización de una clase media en China puede ser un tanto diferente a los que ocurrieron en el contexto europeo.

Desde principios de la década de 1990, he coordinado una serie de entrevistas a empresarios en diferentes lugares de China. Éstas incluyen encuestas en Hangzhou (1991-1993), la provincia de Shanxi (1996-1998, y de nuevo 2000-2002), la provincia de Qinghai (2001-2003), el condado de Jiaocheng, Shanxi (2003-2004), la Ciudad de Qiongshan, Hainan (2004) y

18. David S. G. Goodman, "In Search of China's New Middle Classes: The Creation of Wealth and Diversity", en Asian Studies Review, vol. 22, núm. 1, marzo de 1998, p. 39. 
la Ciudad de Mianyang, provincia de Sichuan (2004-2005). ${ }^{19}$ Se preguntó a los empresarios acerca de sus antecedentes sociales (y de sus familias), sus carreras y actividades empresariales. En términos de formación de la clase media, resaltan tres conclusiones claras en cada entrevista. La primera es que los nuevos empresarios son complejos y no una categoría social simple, incluidos no sólo propietarios-operadores dentro del sector privado, sino muchos gerentes de empresas estatales, colectivas, privadas y extranjeras, además de combinaciones a veces confusas de estas múltiples subcategorías. ${ }^{20} \mathrm{La}$ segunda se relaciona con la riqueza de los nuevos empresarios. Si bien existe una serie de empresarios poco exitosos que debieron enfrentar desastres y otros que únicamente tienen una riqueza moderada, muchos no sólo estaban cómodamente situados respecto a la norma de la economía local cuando se efectuaron las entrevistas, sino que son claramente ricos o súper ricos. Esta conclusión llama la atención hacia los paralelos realizados entre los nuevos empresarios contemporáneos en la RPC y la burguesía europea del siglo XIX. La tercera conclusión general limita estos argumentos al reforzar unos lazos estrechos institucionales y de asociación entre los nuevos empresarios y el partido-Estado: no son independientes ni están excluidos del establecimiento político, el cual, por el contrario, busca activamente incorporarlos.

La noción de cualquier industrialización ab initio en China, desde 1978, se disipa rápidamente al considerar los procesos que generaron a los nuevos empresarios en la era de la reforma. El modelo es el de una persona que tiene una idea, busca capital y establece una empresa para desarrollar una invención

19. Sobre la investigación acerca de Hangzhou (1991-1993) se informa en David S. G. Goodman, "The People's Republic of China: the party-state, capitalist revolution and new entrepreneurs", en R. Robison y D. S. G. Goodman (eds.), The New Rich in Asia: Mobile phones, McDonalds and middle class revolution, Routledge, Londres, 1996, p. 225; acerca de Shanxi (1996-1998) en David S. G. Goodman, "The interdependence of state and society: the political sociology of local leadership", en Chien-min Chao y Bruce J Dickson (eds.), Remarking the Chinese State: Strategies, Society and Security, Routledge, Londres, 2001, p.132; acerca de Shanxi (2000-2), en David S. G. Goodman, "Shanxi as translocal imaginary: reforming the local", en Tim Oakes y Louisa Schein (eds.), Translocal China, Routledge, Londres, 2006, p. 56; acerca de Qinghai (2001-2003), David S. G. Goodman, "Exiled by Definition: The Salar and Economic Activism in Northwest China", en Asian Studies Review, diciembre 2005, vol. 29, núm. 4, pp. 325-343; y acerca de Jiaocheng, Qiongshan y Mianyang, David S. G. Goodman, "Narratives of Change: Culture and local economic development", en B. Krug y H, Hendrischke, China's Economy in the $21^{\text {st }}$ Century, Edward Elgar, 2007.

20. Victor Nee, "Organizational Dynamics of Market Transition: Hybrid Forras, Property Rights, and Mixed Economy in China", en Administrative Science Quarterly, vol. 31, 1992, p.1. 
o innovación; y únicamente se puede aplicar en forma genérica en la RPC. ${ }^{21}$ En general, las nuevas empresas surgidas durante las últimas tres décadas se han generado en una de cuatro formas diferentes, diferenciadas por la fuente de su capital inicial.

Históricamente, la introducción de grandes medidas de determinación del mercado y el desarrollo de nuevos tipos de empresas empezaron en las áreas rurales, con mayor precisión en los distritos rurales, suburbanos y periurbanos de las ciudades. A lo largo de los 1980 y 1990, las empresas de pueblos y ciudades (TVE, Township and Village Enterprises) se volvieron el sostén principal del sector colectivo de la economía y surgieron de las actividades económicas rurales y de las percepciones de la mano de obra existente o de otras formas de capacidad subutilizada. ${ }^{22}$ En el área de Hangzhou, una villa transformó su taller de maquinaria, el cual tenía acceso a productos de alambre, en una línea de producción para utilizar el alambre y producir tarjetas de regalo elaboradas para el mercado japonés. Pronto tuvo tanto éxito que la línea de producción se convirtió en una fábrica a gran escala y la villa dejó su producción agrícola. En Yuci (provincia de Shanxi) otro taller de maquinaria agrícola de la villa se convirtió en una planta productora de radiadores de aluminio; en Yingchuan (también en la provincia de Shanxi), rodeada por minería de carbón, se empezaron a producir subproductos de carbón, especialmente plásticos. Estas empresas y sus desarrollos fueron encauzados por personas de la localidad, con frecuencia el antiguo gerente del taller o cualquier otro nivel de liderazgo local que podía movilizar a sus vecinos. Pese a que técnicamente los gerentes no son los propietarios de las TVE, muchos se comportaban económica, social y políticamente como si lo fueran.

El sector estatal de la economía experimentó procesos similares de desarrollo. El sistema anterior del socialismo estatal se había caracterizado por

21. Barbara Krug (ed.), China's Rational Entrepreneurs: The development of the new private business sector, Routledge, Londres, 2004.

22. Jean C. Oi, State and Peasant in Contemporary China, University of California Press, 1989; Christian Henriot y Shi Lu, La Réforme des Entreprises en Chine: Les entreprises shanghaiennes entre État et marché (Enterprise Reform in China: Shanghai Enterprises between State and Market), L'Harmattan, París, 1996; Lynn T. White, Unstately Power, M. E. Sharpe, White Plains, 1998; Jean C. Oi, Rural China Takes Off Institutional Foundations of Economic Reform University of California Press, 1999; Susan Whiting, Power and Wealth in Rural China: the Political Economy of Institutional Change, Cambridge University Press, 2001; Ray Yep, Manager Empowerment in China: Political implications of rural industrialization in the reform era, Routledge, Londres, 2003. 
ineficiencias a gran escala en la producción y en el sector económico. Con más frecuencia, después de 1984, la reforma económica inevitablemente indujo a los gerentes a que buscaran la eficiencia económica y, en parte como consecuencia, nuevas oportunidades para utilizar los activos que controlaban. En una variedad de formas, los activos del Estado se desarrollaron o incorporaron para producir un beneficio económico. Con frecuencia, las compañías subsidiarias que operaban en el sector colectivo de la economía se establecieron a través de empresas propiedad del Estado o algún departamento de la administración estatal. ${ }^{23}$

En el norte de China, los trabajos del hierro y el acero al inicio de la era de la reforma constituyeron una organización compleja que - al igual que cualquier otra empresa propiedad del Estado a gran escala de ese momentointentó sacar el mayor provecho de las necesidades sociales y de beneficio de su fuerza laboral y de sus dependientes. Las empresas contaban con cafetería comunitaria, tierras agrícolas que abastecían las cafeterías comunitarias y camiones para transportar los productos agrícolas a estas cafeterías comunitarias. Antes de la reforma se puso poca atención al bajo nivel de actividad económica que generaban estas cafeterías comunitarias o al departamento de transporte, ya que cumplían sus tareas asignadas de alimentar a la fuerza laboral y transportar los productos a las cafeterías comunitarias una vez al día. Con la reforma, cada una de estas actividades se dividió y conformó una compañía por separado, técnicamente propiedad de la empresa matriz propiedad del Estado, pero bajo el control de la administración previa a la que se le había asignado una nueva empresa del sector colectivo. Cada una recibió un contrato para suministrar servicios igual que en el pasado, pero ahora para sobrevivir tenían que encontrar trabajo adicional que les generara ingresos en el mercado abierto. En Hangzhou, la ahora famosa compañía de alimentos y bebidas Wahaha se inició a partir de un taller de impresión no lucrativo de una escuela secundaria, cuando sus gerentes se dieron cuenta de que para sobrevivir no sólo debían diversificarse, sino encontrar nuevas actividades económicas.

23. Marc Blecher, "Development State, Entrepreneurial State: The Political Economy of Socialist Reform in Xinju Municipality and Guanghan County", en Gordon White (ed.), The Chinese State in the Era of Economic Reform, Macmillan, Londres, 1991, p. 265; Jane Duckett, The Entrepreneurial State in China, Routledge, 1998; You Ji, China's Enterprise Reform, Routledge, Londres, 1998. 
A pesar de que muchas empresas del sector colectivo fueron establecidas por las empresas matrices propiedad del Estado, la reasignación de los activos del Estado en estas formas a veces también dejó distinciones poco claras entre la propiedad y la gestión. Los gerentes de las empresas del sector estatal que encabezaron la reforma de las compañías claramente quedaron como gerentes, cualquiera que fuera su participación emocional en las empresas de nueva creación. Por otra parte, los gerentes de las empresas del sector colectivo que habían surgido a partir de los activos del Estado, al igual que sus contrapartes semi-rurales, con frecuencia se comportaban en forma similar a la de sus propietarios. En Hangzhou, una vez se le preguntó a un empresario si los activos del Estado que le habían sido efectivamente reasignados a su nuevo estilo de empresa se habían pagado en alguna forma. La respuesta fue muy clara: "No es necesario. Estos fueron antes activos de todo el pueblo y ahora nosotros somos el pueblo".

Ciertamente, existen propietarios-operadores del sector privado que han desarrollado sus propios negocios desde cero con base en una idea innovadora o de una oportunidad en el mercado. ${ }^{24}$ En las diferentes encuestas realizadas desde principios de los 1990, se encontraron emprendedores en todos los sectores y actividades industriales, incluida la minería y la industria pesada, además de la industria ligera, el procesamiento, las ventas al por menor y las industrias de servicios. En general, la mayor parte de los propietariosoperadores del sector privado permanecen en pequeña escala. A medida que crecen sus negocios y desean expandirse, la presión para acceder a los factores de producción: capital de inversión, terreno, mano de obra y permisos políticos, esencialmente indican que un empresario privado exitoso tiene que someter parte de su interés al gobierno local y la constitución como una empresa del sector colectivo. Al igual que en las TVE y aquellas empresas que se desarrollaron a partir del sector y la administración estatales, existe en este tipo de empresas de propietarios-operadores una posible confusión entre propiedad y control.

En Hangzhou, durante los últimos años de la década de los 1980, un técnico de taller descontento dejó su trabajo para abrir su propio taller, calculando

24. Susan Young, Private Business and Economic Reform in China, M. E. Sharpe, Nueva York, 1995; Ross Garnaut y Ligang Song, China's Third Economic Transformation: The Rise of the Private Economy Routledge, Londres, 2003; Bruce Dickson, Red Capitalists: The Party, Private Entrepreneurs, and Prospects for Political Change, Cambridge University Press, 2003. 
que incluso si se reducía su ingreso ganaría más trabajando por su cuenta. Estableció un apiario y produjo miel, la cual vendía él mismo a los restaurantes y hoteles locales. Después de dos años, logró pagar sus deudas y ahorrar cierto capital, de manera que decidió establecer su propio restaurante. Éste a su vez logró ser un gran éxito dejándole después de tres años el capital suficiente para invertir en otra nueva empresa. Pensando que podía moverse hacia el sector de manufactura, buscó un nuevo producto y finalmente decidió establecer una planta de fabricación de procesadores de alimentos. (Había encontrado una máquina que se menciona en la traducción de una novela de Graham Greene y sin saber lo que era, ni investigar más, consideró que podía ser un buen mercado en China.) Desafortunadamente, como empresario privado no tenía acceso a créditos bancarios y la autoridad local le negó el terreno para su fábrica. No fue hasta que aceptó la invitación del gobierno local a cooperar (y ceder la mitad de sus acciones) y crear una nueva empresa del sector colectivo cuando pudo realizar su proyecto.

En el condado de Jiexiu, Shanxi, Li Anmin, ahora presidente y director general de Antai International Enterprise Group Company y uno de los individuos más ricos de la provincia estableció una producción de coque como empresa privada en 1984. Había sido el contador de la villa en su pueblo natal, invirtió 3,000 yuanes de su propio dinero, empleando a 27 de sus vecinos. En los siguientes diez años, la empresa se convirtió en una sociedad anónima colectiva a través de la cooperación con el gobierno local y las acciones de los vecinos de Li. Para mediados de los 1990, la compañía se había expandido a otras actividades, incluido cemento, ropa y ventas al por menor, dando empleo a 3,500 personas. $^{25}$

La cuarta y última forma en la que las nuevas empresas se establecieron fue a través de la inversión extranjera. La penetración de la inversión extranjera se ha incrementado por episodios desde mediados de la década de 1980, dejando relativamente pocas áreas de la economía totalmente restringidas, aunque permanece una alta regulación. Desde la producción de leche en la provincia de Shanxi, a la producción de lana de lujo en la provincia de Qinghai, a la manufactura en Jiaocheng (Shanxi) y Hangzhou, o las ventas al menudeo y servicios en Qiongshan (provincia de Hainan), la inversión extranjera ha tenido lugar en aquellos puntos en los que han surgido oportunidades

25. Liu Liping et al. (eds.) Zhongguo dangdai qlyejla mingdian - Shanxi tao [Contemporary Entrepreneurs in China - Shanxi volume] Beijing, Gongren chubanshe, 1989, p. 302. 
económicas y relaciones con la economía local. Es un tanto confuso que las empresas del sector estatal, las TVE, las empresas del sector colectivo y las compañías privadas hayan establecido operaciones de coinversión con socios en el exterior. Existe igualmente una variedad de relaciones de propiedad atendiendo a estas empresas fundadas por extranjeros. Aunque todas cuentan con gerentes empresarios, en muchos casos no son los originadores de la idea que dio lugar a la cooperación.

\section{Empresarios y riqueza}

Estimar la riqueza de los empresarios en China no es algo fácil. La mayoría de ellos han probado ser un tanto reticentes - como es entendible- a discutir asuntos de su riqueza en una entrevista. Al igual que ocurre con la gente de negocios prácticamente en cualquier lugar del mundo, existe una tendencia a minimizar sus ingresos y ganancias debido a la reglamentación fiscal o tarifaria local. El clima político para los empresarios ciertamente ha mejorado a lo largo de los años y de manera importante desde las primeras entrevistas que se realizaron en 1991, antes de que Deng Xiaoping realizara su "Tour de Inspección del Sur" que reactivó el paso de la reforma económica al año siguiente. ${ }^{26}$ No obstante, la RPC sigue siendo un partido-Estado comunista gobernado por una ideología que - como podemos recordar- siempre ha sido muy rígida, no sólo con los hombres de negocios sino con las personas que se involucran en actividades económicas para beneficio personal. Toda la evidencia disponible podría sugerir que los nuevos empresarios ocupan una posición de riqueza considerable. ${ }^{27}$ Sus patrones de consumo conspicuo ya eran aparentes durante el decenio de 1990, en vivienda, educación privada para sus hijos, alimentos y ropa. ${ }^{28}$ Como anécdota, esto parece haber aumentado sustancialmente desde el año 2000, en especial con el crecimiento de las actividades de ocio en metrópolis como Guangdong, Shanghai, Tianjin y Beijing.

26. David S. G. Goodman, Deng Xiaoping and the Chinese Revolution: A Political Biography Routledge, Londres, 1994, p. 13.

27. Elisabeth Croll, China's New Consumers: Social development and domestic demand, Routledge, Londres, 2006. (Especialmente el capítulo 4 "Elite Lifestyles".)

28. Deborah S. Davis (ed.), The Consumer Revolution in Urban China, University of California Press, 2000, especially Deborah Davis "A Revolution in Consumption", p.1; David Fraser, "Inventing Oasis: Luxury Housing Advertisements and Reconfiguring Domestic Pace in Shanghai", p. 25; y Hanlong Lu "To Be Relatively Comfortable in an Egalitarian Society", p. 124. 
A pesar de que los empresarios entrevistados por lo regular se mostraban renuentes a dar detalles acerca de sus ingresos o ganancias, durante una investigación realizada en Shanxi en 1996-1997, cuatro de cada cinco entrevistados estaban preparados para estimar sus ingresos mensuales en efectivo: sueldo más bonos y pagos adicionales. La tabla 1 muestra en detalle el ingreso estimado promedio ajustado anualmente y diferenciado por categorías de empresario y propiedad empresarial o gestión. Los miembros de las clases profesionales (principalmente médicos y abogados) fueron entrevistados como parte de la misma investigación y también se obtuvo su ingreso promedio anual en efectivo, junto con el ingreso promedio provincial per cápita para 1997.

\section{Tabla 1}

Ingreso en efecto de empresarios: provincia de Shanxi, 1996-1997. Yuan RMB

\begin{tabular}{lc}
\hline Categoría de emprendedor & Ingreso anual en efectivo \\
\hline Gerente, propiedad estatal & 18.627 \\
Gerente, empresa sector colectivo & 17.388 \\
Propietario, empresa privada & 37.200 \\
Gerente, empresa con fondos extranjeros & 18.000 \\
Profesional & 3.700 \\
Ingreso promedio provincial per cápita (1997) & 4.762 \\
\hline
\end{tabular}

Fuente: entrevistas, Shanxi 1996-7 (47 gerentes, empresas propiedad del Estado; 56 gerentes, empresas del sector colectivo; 51 propietarios, empresas privadas; 7 gerentes, empresas con fondos extranjeros, 11 profesionales).

Las diferencias en los ingresos promedio entre las diferentes categorías de nuevos empresarios y personas comunes indicadas en esta tabla son claramente amplias. Los propietarios-operadores dentro del sector privado tienen ingresos en efectivo casi ocho veces superiores al promedio provincial per cápita y en otras categorías de nuevos empresarios, ganan casi cuatro veces el promedio provincial. En Hangzhou, a principios de los años noventa, las diferencias entre los nuevos ricos y el promedio provincial se estimaban en 12:1, de manera que estas relaciones de alguna forma son menores y pueden representar las primeras etapas de desarrollo de la economía en la provincia de Shanxi, que recibió una cantidad considerablemente menor de inversión extranjera y tuvo poco desarrollo industrial. 
Es necesario manejar estas cifras con cuidado, no sólo por los métodos de recopilación de datos, sino también como indicadores de riqueza. Los empresarios con frecuencia cuentan con acceso sin costo (para ellos personalmente) a recursos y reciben subsidios que no están disponibles para otros. Bajo el socialismo de Estado, e incluso en el socialismo de Estado en transición, beneficios similares para alimentos, vivienda, educación, acceso a transporte (especialmente en el uso de coches) y vacaciones también aplican a la mayoría de aquellos que trabajan dentro del partido-Estado. Por ejemplo, es poco probable que los profesionales entrevistados durante 1996-1997 y cuyos ingresos promedio en efectivo se reflejan en la tabla 1 , tuvieran un estándar de vida menor al promedio y muy posiblemente compartieran las aspiraciones de estilo de vida con aquéllos con los que entraron en contacto dentro del partido-Estado, sino también en todas las categorías de nuevos empresarios.

Otra forma de intentar estimar la riqueza de los nuevos empresarios sería considerar los flujos de dinero que controlan y por los cuales son responsables en última instancia. La tabla 2 proporciona los detalles de la información revelada a través de entrevistas con empresarios en Shanxi (1996-1998) acerca de las utilidades empresariales después de impuestos; y en Qiongshan (2004) y Mianyang (2005) respecto a la rotación empresarial anual. Las cifras para cada localidad se contrastan con el ingreso promedio local per cápita correspondiente al momento de las entrevistas. Si bien las cifras que aparecen en la tabla 2 se deben manejar con cuidado, sirven para subrayar la posición clave de los nuevos empresarios en términos de creación de riqueza, además de indicar disparidades considerables, al menos en términos de gestión de riqueza entre los empresarios y el resto de la población.

Por supuesto que no se puede argumentar que todos los empresarios son sumamente ricos. Aquéllos que fueron entrevistados a lo largo de varios años incluyen algunos al borde de la quiebra y al menos uno que en algún momento fue un empresario privado y ha sido afianzado por el gobierno local, debido a que fue un empleador a gran escala cuyo negocio tuvo problemas. Otros simplemente no han tenido éxito u operan un negocio muy modesto. Por lo general, son tiendas minoristas únicas, salones de belleza o actividades industriales de servicio de una sola persona, como las empresas privadas pequeñas que se encuentran prácticamente en todo el mundo. Los promedios que aparecen en las tablas 1 y 2 se derivan de un rango de ganancias y rotación. Las entrevistas realizadas en Shanxi (1996-1997) por ejemplo, encontraron 
un empresario privado cuyo ingreso anual personal en efectivo se estimó en 3,000 yuan RMB y muchos otros cuyos ingresos en efectivo superaron los 70,000 yuan RMB.

Tabla 2

Indicadores de la escala económica:

Utilidades o rotación empresarial promedio. Yuan RMB

\begin{tabular}{|c|c|c|c|c|}
\hline Ubicación & $\begin{array}{l}\text { Fecha de las } \\
\text { entrevistas }\end{array}$ & $\begin{array}{l}\text { Promedio de ingreso } \\
\text { per cápita }\end{array}$ & & \\
\hline \multirow[t]{5}{*}{ Shanxi } & 1996-1998 & 4.762 (1997) & $\begin{array}{l}\text { Utilidades empresariales } \\
\text { promedio después de } \\
\text { impuesto }\end{array}$ & $\begin{array}{l}\text { Millones de } \\
\text { Yuan RMB }\end{array}$ \\
\hline & & & $\begin{array}{l}\text { Empresa } \\
\text { estatal }\end{array}$ & 24,3 \\
\hline & & & Colectividades urbanas & 29,8 \\
\hline & & & Colectividades rurales & 4,2 \\
\hline & & & Empresas privadas & 0,3 \\
\hline Qiongshan & 2004 & 12.697 & $\begin{array}{l}\text { Rotación anual empresarial } \\
\text { promedio }\end{array}$ & 24.0 \\
\hline Mianyang & 2005 & 8.383 & $\begin{array}{l}\text { Rotación anual empresarial } \\
\text { promedio }\end{array}$ & 58,0 \\
\hline
\end{tabular}

Fuente: entrevistas, Shanxi 1996-1998 (230 entrevistados); Qiongshan, 2004 (53 entrevistados); Mianyang, 2005 (56 entrevistados).

\section{Los empresarios y el partido-Estado}

Por cualquier norma, el desarrollo económico en la RPC, ya sea durante la década de los cincuenta o más tarde durante los años ochenta, se ajusta bien al patrón de la industrialización tardía como la que se estableció en Alemania, Japón y Rusia durante finales del siglo XIX. En estos países, el Estado jugó un papel central en la industrialización, en contraposición al capitalismo del laissez-faire de la experiencia europea anterior basada en la protección del papel del individuo fuera del Estado. ${ }^{29}$ Con algunas excepciones, ${ }^{30}$ la mayor parte de la investigación en los cambios socioeconómicos de la reforma en China

29. James Kurth, "Industrial Change and Political Change", en David Collier (ed.), The New Authoritarianism in Latin America, Princeton University Press, 1979, p. 319.

30. Lynn T. White III, Unsiately Power, M. E. Sharpe, White Plains, 1998. 
desde 1978, y en especial la relacionada con la industrialización, ${ }^{31}$ resalta la centralidad del partido-Estado en la generación de cambio. El debate se centra en la medida en la cual el partido-Estado está por sí mismo involucrado en las actividades empresariales, en contraposición a respaldar las actividades económicas de las empresas y los empresarios..$^{32}$

La relación entre los nuevos empresarios y el partido-Estado por lo general es muy cercana en varios aspectos. Muy lejos de ofrecer el marco regulador que ha hecho posible el cambio y los arreglos gubernamentales locales que respaldan ese desarrollo, muchas de las empresas por las que ahora los empresarios son responsables han crecido a partir de actividades del partido-Estado; en donde los empresarios aunque no participaban formalmente en las actividades de dicho partido-Estado, sobre todo en niveles de liderazgo, ahora están totalmente incorporados; $y$ muchos nuevos empresarios parecerían depender de las redes familiares de influencia arraigadas en el partido-Estado. Es notable que estas redes de relaciones e influencia se extiendan incluso entre los empresarios a pequeña escala dentro del sector privado de la economía.

Como se identificó en la descripción de los procesos del desarrollo empresarial durante la era de la reforma, es claro que gran parte de estas nuevas compañías ha surgido ya sea del partido-Estado o están muy cercanas al gobierno local a medida que crecen. Las relaciones de propiedad pueden ser complejas y confusas, pero el crecimiento continuo de las subsidiarias de empresas del sector estatal, las actividades económicas de la administración estatal, así como el sector colectivo cuyo estado teórico fue desafiado hace ya mucho tiempo, aportan amplia evidencia sobre la importancia continua de las relaciones económicas entre los nuevos empresarios y el partido-Estado fuera de un plan formal. Durante la última década, ciertamente es cada vez más claro observar que el sector colectivo de la economía es un sector del gobierno local, en lugar de cómo era en el pasado, parte de la economía estatal de China no totalmente regulada por el proceso de planeación central.

31. Dorothy J. Solinger, From Lathes to Looms: China's Industrial Policy in Comparative Perspective, 1979-1982, Stanford University Press, 1991.

32. Marc Blecher, "Development State, Entrepreneurial State: The Political Economy of Socialist Reform in Xinju Municipality and Guanghan County", en Gordon White (ed.), The Chinese State in the Era of Economic Reform, Macmillan, Londres, 1991, p. 265; Marc Blecher y Vivienne Shue, Tethered Deer: Government and Economy in a Chinese County, Stanford University Press, 1996; Jean C. Oi, Rural China Takes Off: Institutional Foundations of Economic Reform, University of California Press, 1999; Jane Duckett, The Entrepreneurial State in China, Routledge, 1998. 
Una de las consecuencias del crecimiento del sector colectivo es que las filas de los nuevos empresarios contienen a muchos que han venido directamente de las clases medias profesionales y gerenciales. En las entrevistas de la provincia de Shanxi realizadas en 1996-1998, 72\% de los gerentes de empresas estatales entrevistados provenían de una carrera profesional o gerencial, al igual que $79 \%$ de los gerentes de empresas colectivas urbanas, $62 \%$ de los gerentes de empresas colectivas rurales y $42 \%$ de empresas privadas de propietarios-operadores. En las entrevistas de mujeres empresarias en Jiaocheng (2003-2004), 19\% habían sido empleadas previamente en puestos profesionales o gerenciales. Un 19\% de las mujeres empresariales entrevistadas en Qiongshan (2004) tenían antecedentes similares, aunque la cifra en Mianyang (2004-5) representa 36\%.

Como se podría esperar, dadas las dos estructuras del socialismo de Estado y las formas en las que ha ocurrido la formación de empresas, una parte sustancial de los que trabajan en el nivel profesional o gerencial antes de convertirse en empresarios habían sido empleados dentro del partido-Estado. Al mismo tiempo, como se observó, ha sido posible que los empresarios privados se establezcan de manera independiente y desarrollen sus actividades de negocios con éxito antes de que se les requiera cooperar con el gobierno local y otros sectores del partido-Estado si desean mantener una trayectoria de crecimiento. Uno de los costos esperados de los empresarios privados al seguir esta ruta es que asumirán el reto de cargos de liderazgo locales. Con frecuencia, el trato es bastante explícito. Un desarrollador de minas de carbón en Shanxi comentó en la entrevista la forma en que no sólo tuvo que someter la mitad de sus acciones al gobierno local (condado) para poder establecer su empresa (pese a argumentar que desde su punto de vista la compañía seguía siendo privada y de su propiedad) sino que debió unirse al PCC (lo cual había rechazado hasta ese momento) y tener un cargo de liderazgo en el comité del condado del PCC.

El requisito para los nuevos empresarios no siempre es tan formal ni es necesario que asuman un cargo de liderazgo importante dentro del partidoEstado. Ciertamente, ha habido un imperativo creciente para que los empresarios exitosos se unan al PCC y de hecho es uno de los aspectos clave del principio de Jiang Zemin de las "Tres declaraciones", en reconocimiento de que los empresarios deberían ser alentados a unirse al PCC, por primera vez 
desde $1955 .{ }^{33}$ La mayoría de los que empezaron como empresarios privados a pequeña escala y crecieron por la cooperación con el gobierno local, se esperaría después que se unan al PCC, aunque no es necesario que asuman cargos de liderazgo. Al mismo tiempo, existen otras formas en las cuales los nuevos empresarios exitosos son alentados a participar y apoyar al partido-Estado, en especial a través de recibir reconocimiento como empresarios modelo provinciales y nacionales, publicar sus experiencias para su emulación a través de los medios, así como ser elegidos como diputados a los congresos populares del condado, la provincia o la nación. Entre los entrevistados, a muchos se les ha considerado como empresarios modelo y varios fueron electos como diputados a los congresos populares provinciales, mientras dos de ellos son representantes en el congreso popular nacional: un bioquímico de la universidad que regresó a su pueblo natal en Shanxi para establecer una empresa basada en el desarrollo de subproductos del carbón y un hombre que encabezó el desarrollo de la industria del procesamiento de lana de lujo en la provincia de Qinghai.

A pesar de que parecería lógico suponer que muchas de las empresas que se establecieron en la era de la reforma son propiedad o fueron creadas por cuadros líderes, o al menos que los cuadros líderes estuvieron involucrados en el proceso, la evidencia de varias entrevistas realizadas desde 1991 revela que en la mayoría de los casos esto no fue así. Si bien se puede concebir que los métodos de selección de los entrevistados podrían haber impedido en gran medida la posibilidad de encontrar a cuadros líderes como empresarios, el tiempo invertido en el campo en un lugar dado y el grado de familiaridad desarrollado probablemente hubiera aportado información sobre las actividades de ese tipo en caso de que hubieran existido. Sin embargo, no se generó ningún tipo de evidencia anecdótica sobre el particular.

Aunque al mismo tiempo, parecería que los vínculos familiares con los cuadros líderes en el partido-Estado son importantes también en el proceso de formación de las empresas. La evidencia de las entrevistas de la provincia de Shanxi realizadas en 1996-1998, señala que existe un claro patrón de tres generaciones. Los cuadros líderes en su totalidad se reclutan por méritos intelectuales entre el pueblo, como ha sido la práctica del PCC en la República Popular China. Realizan sus trabajos y se retiran, pero sus hijos se convierten en gente de negocios, aprovechando en especial las relaciones locales y las

33. Anunciado por Jiang en el Decimosexto Congreso CCP en 2002. 
redes de influencia creadas por sus padres. ${ }^{34}$ Como comentó un empresario que desarrolló una planta de hierro y acero mediana en un campo en la localidad en donde su padre fue secretario del PCC durante varios años, cuando se le preguntó por qué se unió a uno de los partidos políticos democráticos patrocinados por el estado y no al PCC: “¿Por qué debería unirme al PCC? Mi padre es el dueño del pueblo".

Ciertamente, todas las entrevistas sugieren de manera importante que si bien no es necesario que los nuevos emprendedores sean miembros del PCC, es sumamente útil que sus familias tengan miembros en el PCC y hayan formado parte del partido-Estado. Incluso aunque las muestras elegidas para las entrevistas no sean directamente representativas de la población como un todo, o de los empresarios de cada localidad necesariamente, las proporciones cuyas familias se vinculan a las diferentes redes de influencia de este tipo son demasiado altas para ser ignoradas. En la actualidad cerca de 5.4\% de la población total de China es miembro del PCC, aunque la proporción fue un tanto menor durante el decenio de 1990.

En las entrevistas realizadas en Shanxi, entre 1996-1998, 60\% de los propietarios-operadores de empresas privadas no eran miembros del PCC, pero $39 \%$ tenían al menos un padre que lo había sido; $35 \%$ de los gerentes de empresas del sector colectivo no eran miembros del PCC, pero de nuevo $39 \%$ tenían al menos un padre que lo había sido. En otras entrevistas hay proporciones similares. En Jiaocheng, en 2003-2004, únicamente $24 \%$ de los empresarios eran miembros del PCC o tenían una conexión directa con el partido-Estado, en contraposición a 42\% de sus padres. En Qiongshan (2004) es similar, únicamente que $23 \%$ de los entrevistados eran miembros del PCC o habían tenido alguna relación directa con el partido-Estado, en comparación con $40 \%$ de sus padres. En las entrevistas de Mianyang (2004-5) las proporciones fueron un poco más altas: $35 \%$ para los entrevistados miembros del PCC o directamente involucrados con el partido-Estado, mientras que 50\% de sus padres lo eran. En Jiaocheng las entrevistas mostraron que $58 \%$ de las participantes tenían un padre o suegro (las empresarias entrevistadas eran todas mujeres) que eran miembros del PCC, en Qiongshan la proporción fue de $55 \%$ y en Mianyang de $66 \%$.

34. David S. G. Goodman, "The Localism of Local Leadership: Cadres in Reform Shanxi”, en Journal of Contemporary China, vol. 9, núm. 24, 2000, p. 159. 


\section{Conclusión: nuevos empresarios y clase media}

Inevitablemente el cambio social en China es un tanto sui generis como evocación de los procesos ocurridos en otros sistemas políticos. Las complicaciones, desde la perspectiva de la comparación, para analizar a la República Popular China consisten en que el punto de partida para el cambio reciente ha sido un sistema socialista de Estado de industrialización tardía que fue elegido para introducir las reformas de mercado. La propiedad, la gestión y el control están entretejidos de tal manera que cruzan los análisis anteriores de los comportamientos de la clase media (o incluso cualquier otra).

Por un lado, los nuevos empresarios no son una nueva clase media debido a que durante mucho tiempo han sido una clase profesional y gerencial establecida. Si bien proceden de antecedentes gerenciales y ocupan puestos que giran alrededor del control de las empresas, muchos tienen un involucramiento emotivo mucho mayor en su puesto personal en las empresas que lo implicado por este término en general. Tampoco en la mayoría de los casos los nuevos empresarios representan las secciones de ingresos medios de la población. Por otro lado, en la medida en que la creciente clase media pueda contribuir con el desarrollo económico para convertirse en el eje central de la clase gobernante, entonces es posible ver un proceso similar en formación en la RPC. La diferencia, por supuesto, es que la RPC sigue siendo un partidoEstado comunista y los nuevos empresarios, lejos de ser excluidos, han sido invitados a incorporarse en el establecimiento político.

Estas observaciones resaltan los objetivos de los diferentes discursos de las clases medias que se encuentran tanto dentro de China como en el exterior. En China existe una limitación ideológica clara para fomentar y generar el crecimiento económico de la riqueza, incluso a medida que la tercera década de estas políticas llega a su fin. Promover a los nuevos empresarios y nuevos ricos como las nuevas clases medias es algo más igualitario y ciertamente más aceptable en general que describirlos como los súper ricos o la nueva burguesía.

Alentar el desarrollo de las nuevas clases medias comúnmente también es una forma de promover la iniciativa individual y un crecimiento económico autoimpulsado, igual que la creación de riqueza. En una sociedad como un todo, quizá es más cómodo y generalmente mejor aceptado que las personas tengan aspiraciones de clase media más que convertirse en una megaestrella de la lista A. En realidad, según se describe específicamente, las aspiraciones de 
la clase media se pueden representar y vincular con la modernización y estilos de vida deseables a través de los medios masivos de comunicación.

Fuera de China, a veces parece que aquellos que intentan interpretar su desarrollo, realmente desean ser capaces de encontrar a la clase media en su cambio social contemporáneo. Si bien una explicación completa del fenómeno se encuentra en otro sitio, una posibilidad clara es que la transformación de la República Popular China luce esperanzadora para aquellos que ven una ecuación entre la industrialización y el desarrollo económico por un lado y el surgimiento de una democracia liberal que asegure la paz, por el otro. El argumento de que son gente "como uno" es muy seductor, especialmente si se ofrece con un toque de ironía. my 